\title{
Research Article \\ Results for Twin Singular Nonlinear Problems with Damping Term
}

\author{
Youwei Zhang \\ Department of Mathematics, Hexi University, Gansu 734000, China \\ Correspondence should be addressed to Youwei Zhang,ywzhang0288@163.com
}

Received 6 December 2010; Accepted 7 March 2011

Academic Editor: Wolfgang Castell

Copyright (c) 2011 Youwei Zhang. This is an open access article distributed under the Creative Commons Attribution License, which permits unrestricted use, distribution, and reproduction in any medium, provided the original work is properly cited.

By using the functional type cone expansion and compression fixed-point theorem in cones, some new and general results on the existence of positive solution for twin singular boundary value problems with damping term are obtained. An example is given to illustrate our results.

\section{Introduction}

The study of multipoint boundary value problem for linear second-order ordinary differential equation was initiated by I1'in and Moiseev [1] motivated by the work of Bitsadze and Samarskir [2-4] on nonlocal linear elliptic boundary value problem, which is a new area of still fairly theoretical exploration in mathematics. Several authors have expounded on various aspects of this theory; see the survey paper by Gupta et al. [5-7] and the references cited therein. Thereamong, the study of singular boundary value problem for ordinary differential equation has led to several important applications in applied mathematics and physical science, such as the Thomas-Fermi problem

$$
\begin{gathered}
x^{\prime \prime}(t)-t^{-1 / 2} x^{3 / 2}=0, \quad 0<t<1, \\
x(0)=0=x(1),
\end{gathered}
$$

which appears in determining the electrical potential in an atom. For other application results, we refer to [8-10]. With regards to this, increasing attention is paid to question of singular boundary value problem and has obtained many excellent results of the existence of the positive solution for two multiple points nonlinear singular boundary value problem [11-15]. The main techniques are the upper and lower solutions method [16], the LeraySchauder continuation theory [17], and the fixed-point theory in cones [18] et al. We would 
like to mention some results of Ma and O'Regan [13] and Yao [15], which motivated us to consider the singular boundary value problems. In [13], the authors have studied a multipoint boundary value problem

$$
\begin{gathered}
x^{\prime \prime}(t)=f\left(t, x(t), x^{\prime}(t)\right)+e(t), \quad 0<t<1, \\
x^{\prime}(0)=0, \quad x(1)=\sum_{i=1}^{m-2} a_{i} x\left(\xi_{i}\right),
\end{gathered}
$$

where $\left.\xi_{i} \in\right] 0,1\left[, a_{i} \in \mathbb{R}, i=1,2, \ldots, m-2,0<\xi_{1}<\xi_{2}<\cdots<\xi_{m-2}<1, f:[0,1] \times \mathbb{R}^{2} \rightarrow \mathbb{R}\right.$ is a function satisfying Carathéodory's conditions and $\left.(1-t) e(t) \in L^{1}\right] 0,1[$; the Leray-Schauder continuation theorem leads to the existence of single $C^{1}[0,1$ [ solution. The literature [15] has discussed a second-order boundary value problem

$$
\begin{gathered}
x^{\prime \prime}(t)+h(t) f(x(t))=0, \quad 0<t<1, \\
a x(0)-b x^{\prime}(0)=0, \quad a x(1)+b x^{\prime}(1)=0,
\end{gathered}
$$

where $h(t)$ is symmetric on ]0,1[ and may be singular at both end points $t=0$ and $t=$ 1 . The author has proved the existence of $n$ symmetric positive solutions and established a corresponding iterative scheme, the main tool being the monotone iterative technique.

A powerful tool for proving existence of solution to boundary value problem is the fixed-point theory. In many cases, it is possible to find single, double, or multiple solutions for boundary value problem, and for the same problem, using the various methods, one can obtain different results under some appropriate conditions. To my best knowledge, very little work has been done on the existence of positive solution for boundary value problem by using the functional type cone expansion and compression fixed-point theorem. The aim of this paper is to establish some new and general results on the existence of positive solution to singular boundary value problems with damping term

$$
\begin{gathered}
u^{\prime \prime}(t)-\lambda u^{\prime}(t)+h(t) f(t, u(t))=0, \quad a<t<b, \\
u^{\prime}(a)-\lambda u(a)=0, \quad \gamma u(b)+\delta u^{\prime}(b)=\sum_{i=1}^{n} a_{i} u\left(t_{i}\right), \\
\gamma u(a)+\delta u^{\prime}(a)=\sum_{i=1}^{n} a_{i} u\left(t_{i}\right), \quad u^{\prime}(b)-\lambda u(b)=0,
\end{gathered}
$$

where $\lambda, \gamma, \delta, a_{i}, t_{i},(i=1,2, \ldots, n), h$, and $f$ satisfy

$\left.\left(\mathrm{H}_{1}\right)-\infty<a<t_{1}<\cdots<t_{n}<b<\infty, \lambda, \gamma, \delta \in\right] 0, \infty\left[, a_{i} \in\right] 0, \infty[,(i=1,2, \ldots, n)$,

$\left(\mathrm{H}_{2}\right) d_{1}=(\gamma+\lambda \delta) \exp (\lambda b)-\sum_{i=1}^{n} a_{i} \exp \left(\lambda t_{i}\right)>0, d_{2}=\sum_{i=1}^{n} a_{i} \exp \left(\lambda t_{i}\right)-(\gamma+\lambda \delta) \exp (\lambda a)>0$,

$\left.\left(\mathrm{H}_{3}\right) h:\right] a, b[\rightarrow[0, \infty[$ is continuous function, $h(t)$ may be singular at $t=a$ and $/$ or $t=b$, and $0<\int_{a}^{b} h(s) d s<\infty$,

$\left(\mathrm{H}_{4}\right) f:[a, b] \times[0, \infty[\rightarrow[0, \infty[$ satisfies the Carathéodory condition, that is, for each $x \in[0, \infty[$, the mapping $t \rightarrow f(t, x)$ is Lebesgue measurable on $[a, b]$ and for a.e. $t \in[a, b]$, the mapping $x \rightarrow f(t, x)$ is continuous on $[0, \infty[$, and for each $r>0$, there exists $\phi_{r} \in L^{1}[a, b]$ such that $f(t, x) \leq \phi_{r}(t)$ for all $u \in[0, r]$ and for a.e. $t \in[a, b]$. 
We will impose some advisable conditions on the nonlinearity $f$ to ensure the existence of at least one positive solution for the above problems. In order to obtain our results, we construct special operator which is the base for further discussion and provide two crucial functionals on cones. Applying the functional type cone expansion and compression fixedpoint theorem to the operator and functionals, we obtain some new and general results on the existence of at least one positive solution for the twin singular problems (1.4), (1.5) and (1.4), (1.6). Our results improve and generalize those in $[15,19]$.

Let $\alpha$ and $\beta$ be nonnegative continuous functionals on a cone $D$ in real Banach space $B$. For positive numbers $r$ and $L$, we define the sets

$$
\begin{gathered}
D(\alpha, r)=\{x \in P: r<\alpha(x)\}, \\
D(\beta, L)=\{x \in P: \beta(x)<L\}, \\
P(\beta, \alpha, r, L)=\{x \in P: r<\alpha(x), \beta(x)<L\} .
\end{gathered}
$$

We state the functional type cone expansion and compression fixed-point theorem [20].

Lemma 1.1. Let $D$ be a cone in a real Banach space $B$, and let $\alpha$ and $\beta$ be nonnegative continuous functionals on $D$. Let $P(\beta, \alpha, r, L)$ be a nonempty bounded subset of $D$,

$$
\tau: \overline{p(\beta, \alpha, r, L)} \longrightarrow p
$$

is a completely continuous operator with

$$
\inf _{x \in \partial p(\beta, \alpha, r, L)}\|\tau x\|>0, \quad \overline{p(\alpha, r)} \subseteq p(\beta, L)
$$

for $p(\beta, L)$. If $\alpha(\tau x) \geq r$ for all $x \in \partial p(\alpha, r), \beta(\tau x) \leq L$ for each $x \in \partial p(\beta, L)$, and for each $y \in \partial P(\alpha, r), z \in \partial P(\beta, L), \theta \in] 0,1]$, and $\mu \in[1, \infty[$, the functionals satisfy the properties

$$
\alpha(\theta y) \leq \theta \alpha(y), \quad \beta(\mu z) \geq \mu \beta(z), \quad \beta(0)=0,
$$

then $\tau$ has at least one positive fixed-point $x$ such that

$$
r \leq \alpha(x), \quad \beta(x) \leq L
$$

\section{Main Results}

Let $B$ be the Banach space $C^{1}[a, b]$ with the norm $\|u\|=\max \left\{\|u\|_{0},\left\|u^{\prime}\right\|_{1}\right\}$, where $\|u\|_{0}=$ $\sup _{t \in[a, b]}|u(t)|$ and $\left\|u^{\prime}\right\|_{1}=\sup _{t \in] a, b[}\left|\mathcal{u}^{\prime}(t)\right|$, and let a cone in $B$

$$
p_{1}=\{v \in \mathbb{B}: v \text { is nondecreasing on }[a, b], v(a)=0\}
$$


For $v \in p_{1}$, define the operator $\tau_{1}$ by

$$
\tau_{1} v(t)=\int_{a}^{t} h(s) f\left(s, \exp (\lambda s)\left(c_{1}(v)+\int_{s}^{b} v(\omega) \exp (-\lambda \omega) d \omega\right)\right) d s, \quad t \in[a, b],
$$

where $c_{1}(v)=\left(1 / d_{1}\right)\left(\delta v(b)+\sum_{i=1}^{n} a_{i} \exp \left(\lambda t_{i}\right) \int_{t_{i}}^{b} v(\omega) \exp (-\lambda \omega) d \omega\right)$.

Lemma 2.1. If $v \in p_{1}$ is a fixed-point $\tau_{1}$, then

$$
u(t):=\exp (\lambda t)\left(c_{1}(v)+\int_{t}^{b} v(\omega) \exp (-\lambda \omega) d \omega\right)
$$

is one solution of the problem (1.4), (1.5).

Proof. Suppose that $v \in p_{1}$ is a fixed-point $\tau_{1}$ and $u(t)=\exp (\lambda t)\left(c_{1}(v)+\int_{t}^{b} v(\omega) \exp (-\lambda \omega) d \omega\right)$, thus we have

$$
u^{\prime}(t)-\lambda u(t)=-v(t)=-\tau_{1} v(t)=-\int_{a}^{t} h(s) f\left(s, \exp (\lambda s)\left(c_{1}(v)+\int_{s}^{b} v(\omega) \exp (-\lambda \omega) d \omega\right)\right) d s
$$

Further,

$$
u^{\prime \prime}(t)-\lambda u^{\prime}(t)=-h(t) f\left(t, \exp (\lambda t)\left(c_{1}(v)+\int_{t}^{t_{n}} v(\omega) \exp (-\lambda \omega) d \omega\right)\right)=-h(t) f(t, u(t))
$$

The boundary condition (1.5) is satisfied due to the relation between $u, v$, and $c_{1}(v)$.

For $v \in p_{1}$, we define the nonnegative continuous functionals $\alpha$ and $\beta$ on $p_{1}$ by

$$
\alpha(v)=\int_{t_{1}}^{b} v(\omega) \exp (-\lambda \omega) d \omega, \quad \beta(v)=v\left(t_{1}\right)
$$

Lemma 2.2. Let $r_{1}>0$. If $v \in \partial p_{1}\left(\alpha, r_{1}\right)$, then

$$
\begin{gathered}
v\left(t_{1}\right) \leq \frac{\exp (\lambda b)}{b-t_{1}} r_{1}, \\
\int_{a}^{b} v(\omega) \exp (-\lambda \omega) d \omega \geq r_{1} .
\end{gathered}
$$


Proof. For $v \in \partial p_{1}\left(\alpha, r_{1}\right)$, that is, $\alpha(v)=r_{1}$. Since $v(t)$ nondecreasing on $\left[a, t_{1}\right]$, we have

$$
r_{1}=\alpha(v)=\int_{t_{1}}^{b} v(\omega) \exp (-\lambda \omega) d \omega \leq \int_{t_{1}}^{b} v(b) \exp (-\lambda \omega) d \omega \leq v(b) \exp \left(-\lambda t_{1}\right)\left(b-t_{1}\right)
$$

so we get (2.7). Furthermore,

$$
\int_{a}^{b} v(\omega) \exp (-\lambda \omega) d \omega=\int_{a}^{t_{1}} v(\omega) \exp (-\lambda \omega) d \omega+\int_{t_{1}}^{b} v(\omega) \exp (-\lambda \omega) d \omega \geq r_{1}
$$

Lemma 2.3. Let $L_{1}>0$. If $v \in \partial p_{1}\left(\beta, L_{1}\right)$, then

$$
\int_{t_{1}}^{b} v(\omega) \exp (-\lambda \omega) d \omega \geq L_{1} \exp (-\lambda b)\left(b-t_{1}\right)
$$

Proof. For $v \in \partial p_{1}\left(\beta, L_{1}\right)$, that is to say, $\beta(v)=L_{1}$. In view of $v(t)$ is nondecreasing on $[a, b]$, for $\omega \in\left[t_{1}, b\right]$, we have

$$
v(\omega) \geq v\left(t_{1}\right)=\beta(v)=L_{1} .
$$

Hence,

$$
\int_{t_{1}}^{b} v(\omega) \exp (-\lambda \omega) d \omega \geq L_{1} \int_{t_{1}}^{b} \exp (-\lambda \omega) d \omega \geq L_{1} \exp (-\lambda b)\left(b-t_{1}\right)
$$

Lemma 2.4. Let $\left(H_{1}\right)-\left(H_{4}\right)$ hold, then $\tau_{1}: p_{1} \rightarrow p_{1}$ is completely continuous.

Proof. By $\left(H_{1}\right)-\left(H_{4}\right)$, we observe that $\tau_{1} v \in C^{1}[a, b],\left(\tau_{1} v\right)(t)$ is nondecreasing on $[a, b]$, and $\left(\tau_{1} v\right)(a)=0$, so $\tau_{1}: p_{1} \rightarrow p_{1}$. Since $h(t)$ may be singular at $t=a$ and /or $t=b$, we take the arguments to show that $\tau_{1}$ is completely continuous.

Assume that $v_{n}, v_{0} \in p_{1}$. In view of $f$ satisfying the Carathéodory condition, it is easy to see that

$$
\left\|v_{n}-v_{0}\right\|_{0} \longrightarrow 0 \text { implies that }
$$

$$
\begin{aligned}
\sup _{s \in \Omega} \mid f\left(s, \exp (\lambda s)\left(c_{1}\left(v_{n}\right)+\int_{s}^{b} v_{n}(\omega) \exp (-\lambda \omega) d \omega\right)\right) \\
\quad-f\left(s, \exp (\lambda s)\left(c_{1}\left(v_{0}\right)+\int_{s}^{b} v_{0}(\omega) \exp (-\lambda \omega) d \omega\right)\right) \mid d s \longrightarrow 0
\end{aligned}
$$


as $n \rightarrow \infty$, where $\Omega=[a, b]$ or $] a, b[$. Thus, we have

$$
\begin{aligned}
\left\|\tau_{1} v_{n}-\tau_{1} v_{0}\right\|_{0}= & \sup _{t \in[a, b]}\left|\left(\tau_{1} v_{n}\right)(t)-\left(\tau_{1} v_{0}\right)(t)\right| \\
\leq \int_{a}^{b} h(s) \mid f\left(s, \exp (\lambda s)\left(c_{1}\left(v_{n}\right)+\int_{s}^{b} v_{n}(\omega) \exp (-\lambda \omega) d \omega\right)\right) & \quad f\left(s, \exp (\lambda s)\left(c_{1}\left(v_{0}\right)+\int_{s}^{b} v_{0}(\omega) \exp (-\lambda \omega) d \omega\right)\right) \mid d s, \\
\left\|\left(\tau_{1} v_{\mathrm{n}}\right)^{\prime}-\left(\tau_{1} v_{0}\right)^{\prime}\right\|_{1}=\sup _{t \in], b[}\left|\left(\tau_{1} v_{n}\right)^{\prime}(t)-\left(\tau_{1} v_{0}\right)^{\prime}(t)\right| & \left.\quad-f\left(t, \exp (\lambda t)\left(c_{1}\left(v_{0}\right)+\int_{s}^{b} v_{0}(\omega) \exp (-\lambda \omega) d \omega\right)\right) \mid\right) .
\end{aligned}
$$

Therefore,

$$
\left\|\tau_{1} v_{n}-\tau_{1} v_{0}\right\| \longrightarrow 0 \quad(n \longrightarrow \infty)
$$

This means that the operator $\tau_{1}: p_{1} \rightarrow p_{1}$ is continuous.

Choose two sequences $\left.\left\{\varphi_{n}\right\}_{n=1}^{\infty},\left\{\psi_{n}\right\}_{n=1}^{\infty} \subset\right] a, b$ [ satisfying $\varphi_{n} \leq \psi_{n}$ for any $n \geq 1$, such that $\varphi_{n} \rightarrow a$ and $\psi_{n} \rightarrow b$ as $n \rightarrow \infty$, respectively. Define

$$
h_{n}(t)= \begin{cases}\inf _{a \leq t \leq \varphi_{n}} h(t), & \\ h(t), & \varphi_{n}<t<\psi_{n} \\ \inf _{\psi_{n} \leq t \leq b} h(t), & \end{cases}
$$

and an operator sequence $\left\{\tau_{1 n}\right\}_{n=1}^{\infty}$ by

$$
\tau_{1 n} v=\int_{a}^{t} h_{n}(s) f\left(s, \exp (\lambda s)\left(c_{1}(v)+\int_{s}^{b} v(\omega) \exp (-\lambda \omega) d \omega\right)\right) d s
$$

Clearly, $h_{n}:[a, b] \rightarrow[0, \infty)$ is a piecewise continuous function, and the operator $\tau_{1 \mathrm{n}}: p_{1} \rightarrow$ $p_{1}$ is well defined. Further, we can see that $\tau_{1 n}: p_{1} \rightarrow p_{1}$ is completely continuous.

Let $R>0, B_{R}:=\left\{v \in p_{1}:\|v\|_{0} \leq R\right\}$, and $M_{R}=\sup \{f(t, u):(t, u) \in[a, b] \times[0, \bar{R}]\}$, where $\bar{R}=\left(R \exp (\lambda b) / d_{1}\right)\left(\delta+(1 / \lambda) \sum_{i=1}^{n} a_{i}\left(1-\exp \left(-\lambda\left(b-t_{i}\right)\right)\right)\right)+(R / \lambda)(\exp \lambda(b-a)-1)>0$. 
We will prove that $\tau_{1 n}$ approach $\tau_{1}$ uniformly on $B_{R}$. From the absolute continuity of integral, we obtain

$$
\lim _{n \rightarrow \infty} \int_{l(n)} h(s) d s=0
$$

where $l(n)=\left[a, \varphi_{n}\right] \cup\left[\psi_{n}, b\right]$. For each $v \in B_{R}, t \in\left[a, \varphi_{n}\right]$, we have

$$
\begin{aligned}
\left\|\tau_{1 n} v-\tau_{1} v\right\|_{0} & =\sup _{t \in\left[a, \varphi_{n}\right]} \mid \int_{a}^{t}\left(h_{n}(s)-h(s)\right) f\left(s, \exp (\lambda s)\left(c_{1}(v)+\int_{s}^{b} v(\omega) \exp (-\lambda \omega) d \omega\right)\right) d s \\
& \leq M_{R} \int_{a}^{\varphi_{n}}\left|h_{n}(s)-h(s)\right| d s \longrightarrow 0 \quad(n \longrightarrow \infty) .
\end{aligned}
$$

For each $v \in B_{R}, t \in\left[\psi_{n}, b\right]$, we have

$$
\begin{aligned}
\left\|\tau_{1 n} v-\tau_{1} v\right\|_{0} & =\sup _{t \in\left[\psi_{n}, b\right]} \mid \int_{a}^{t}\left(h_{n}(s)-h(s)\right) f\left(s, \exp (\lambda s)\left(c_{1}(v)+\int_{s}^{b} v(\omega) \exp (-\lambda \omega) d \omega\right)\right) d s \\
& \leq M_{R} \int_{a}^{b}\left|h_{n}(s)-h(s)\right| d s \longrightarrow 0 \quad(n \longrightarrow \infty) .
\end{aligned}
$$

It is easy to see that, for each $v \in B_{R}$ and $\varphi_{n}<t<\psi_{n}$, there is $\left\|\tau_{1 n} v-\tau_{1} v\right\|_{0} \rightarrow 0$ as $n \rightarrow \infty$. Similarly, for any $v \in B_{R}$, and $\left.t \in\left[a, \varphi_{n}\right],\right] \varphi_{n}, \psi_{n}\left[,\left[\psi_{n}, b\right]\right.$, respectively, we can obtain that

$$
\begin{aligned}
&\left\|\left(\tau_{1 n} v\right)^{\prime}-\left(\tau_{1} v\right)^{\prime}\right\|_{1}=\sup _{t}\left|\left(h_{n}(t)-h(t)\right) f\left(t, \exp (\lambda t)\left(c_{1}(v)+\int_{t}^{b} v(\omega) \exp (-\lambda \omega) d \omega\right)\right)\right| \\
& \leq M_{R}\left|h_{n}(t)-h(t)\right| \longrightarrow 0 \quad(n \longrightarrow \infty) .
\end{aligned}
$$

From the above argument, we obtain

$$
\left\|\tau_{1 n} v-\tau_{1} v\right\|=\max \left\{\left\|\tau_{1 n} v-\tau_{1} v\right\|_{0},\left\|\left(\tau_{1 n} v\right)^{\prime}-\left(\tau_{1} v\right)^{\prime}\right\|_{1}\right\} \rightarrow 0 \quad(n \longrightarrow \infty)
$$

That is to say, the sequence $\tau_{1 n}$ is uniformly approximate $\tau_{1}$ on any bounded subset of $p_{1}$. Therefore, $\tau_{1}: p_{1} \rightarrow p_{1}$ is completely continuous. 
For convenience, we set

$$
\begin{gathered}
m_{1}=\int_{t_{1}}^{b} \exp (-\lambda t)\left(\int_{a}^{t} h(s) d s\right) d t, \quad M_{1}=\int_{a}^{b} h(s) d s, \\
u_{1}=r_{1} \exp (\lambda a)\left(\frac{\delta \exp (\lambda a)}{d_{1}(b-a)}+1\right), \quad u_{2}=L_{1} \exp \left(\lambda t_{1}\right) \frac{\delta+\sum_{i=1}^{n} a_{i}\left(b-t_{i}\right) \exp \left(\lambda\left(t_{i}-b\right)\right)}{d_{1}} .
\end{gathered}
$$

We are now ready to apply a functional type cone expansion and compression fixedpoint theorem to the operator $\tau_{1}$ to give the sufficient conditions for the existence of at least one positive solution to the problem (1.4), (1.5).

Theorem 2.5. Suppose that $\left(H_{1}\right)-\left(H_{4}\right)$ hold. Assume that there exist positive numbers $k_{1}, r_{1}$, and $L_{1}$ with $(\exp (\lambda b)) /\left(b-t_{1}\right) r_{1}<L_{1}$ such that

$$
\begin{aligned}
& \left(\mathrm{A}_{1}\right) f(t, w) \geq k_{1},(t, w) \in[a, b] \times\left[r_{1}, \infty[\right. \\
& \left(\mathrm{A}_{2}\right) f(t, w) \geq r_{1} / m_{1},(t, w) \in\left[a, t_{1}\right] \times\left[u_{1}, \infty[\right. \\
& \left(\mathrm{A}_{3}\right) f(t, w) \leq L_{1} / M_{1},(t, w) \in\left[t_{1}, b\right] \times\left[u_{2}, \infty[\right.
\end{aligned}
$$

then the operator $\tau_{1}$ has at least one fixed-point $v$ such that $r_{1} \leq \alpha(v)$ and $\beta(v) \leq L_{1}$, and the problem (1.4), (1.5) has at least one positive solution $u$ such that

$$
u(t)=\exp (\lambda t)\left(c_{1}(v)+\int_{t}^{b} v(\omega) \exp (-\lambda \omega) d \omega\right)
$$

Proof. The cone $p_{1}$ and operator $\tau_{1}$ are defined by (2.1) and (2.2), respectively. By the properties of operator $\tau_{1}$, it suffices to show that the conditions of Lemma 1.1 hold with respect to $\tau_{1}$. In view of Lemma 2.1, it is not difficult to prove that a fixed point of $\tau_{1}$ is coincident with the solution of the boundary value problem (1.4), (1.5), so we concentrate on the existence of the fixed point of the operator $\tau_{1}$. Set $p_{1}\left(\beta, \alpha, r_{1}, L_{1}\right)$ is a nonempty bounded subset of $p_{1}$. From Lemma 2.4, it can be shown that

$$
\tau_{1}: \overline{p_{1}\left(\beta, \alpha, r_{1}, L_{1}\right)} \longrightarrow p_{1}
$$

is completely continuous by the Arzela-Ascoli lemma. For $v \in \partial p_{1}\left(\beta, \alpha, r_{1}, L_{1}\right)$, the assumption $\left(\mathrm{A}_{1}\right)$ implies that

$$
\begin{aligned}
& \left\|\tau_{1} v\right\|_{0}=\int_{a}^{b} h(s) f\left(s, \exp (\lambda s)\left(c_{1}(v)+\int_{s}^{b} v(\omega) \exp (-\lambda \omega) d \omega\right)\right) d s \geq k_{1} \int_{a}^{b} h(s) d s, \\
& \left\|\left(\tau_{1} v\right)^{\prime}\right\|_{1}=\sup _{t \in] a, b[} h(t) f\left(t, \exp (\lambda t)\left(c_{1}(v)+\int_{t}^{b} v(\omega) \exp (-\lambda \omega) d \omega\right)\right) \geq k_{1} \sup _{t \in] a, b[} h(t) .
\end{aligned}
$$


the hypotheses of $\left(\mathrm{H}_{3}\right)$ lead to

$$
\inf _{v \in \partial p_{1}(\beta, \alpha, r, L)}\left\|\tau_{1} v\right\| \geq k_{1} \min \left\{\sup _{t \in] a, b[} h(t), \int_{a}^{b} h(s) d s\right\}>0 .
$$

If $v \in \overline{p_{1}\left(\alpha, r_{1}\right)}$, then $\beta(v)=v\left(t_{1}\right) \leq(\exp (\lambda b)) /\left(b-t_{1}\right) r_{1}<L_{1}$, and so $v \in \overline{p_{1}\left(\beta, L_{1}\right)}$, that is, $\overline{p_{1}\left(\alpha, r_{1}\right)} \subseteq p_{1}\left(\beta, L_{1}\right)$. It follows that the conditions of Lemma 1.1 hold with respect to $\tau_{1}$. By the definition of functionals $\alpha$ and $\beta$, we can check that the functionals satisfy the properties $\alpha(\theta y)=\int_{t_{1}}^{b} \theta y(\omega) \exp (-\lambda \omega) d \omega=\theta \int_{t_{1}}^{b} y(\omega) \exp (-\lambda \omega) d \omega=\theta \alpha(y)$ for $y \in \partial p_{1}\left(\alpha, r_{1}\right)$ and $\theta \in] 0,1], \beta(\mu z) \stackrel{=}{=} \mu\left(t_{1}\right)=\mu \beta(z)$ for $z \in \partial p_{1}\left(\beta, L_{1}\right)$ and $\mu \in[1, \infty[, \beta(0)=0$.

We now prove that $\alpha\left(\tau_{1} v\right) \geq r_{1}$, in Lemma 1.1, holds. In fact, if $v \in \partial p_{1}\left(\alpha, r_{1}\right)$, by the properties of $c_{1}(v)$ and Lemma 2.2, for each $t \in\left[a, t_{1}\right]$,

$$
\begin{aligned}
u(t) & =\exp (\lambda t)\left(c_{1}(v)+\int_{t}^{b} v(\omega) \exp (-\lambda \omega) d \omega\right) \\
& \geq \exp (\lambda a)\left(c_{1}(v)+r_{1}\right) \\
& \geq r_{1} \exp (\lambda a)\left(\frac{\delta \exp (\lambda a)}{d_{1}(b-a)}+1\right) .
\end{aligned}
$$

Hence, by the assumption $\left(A_{2}\right)$ and $(2.28)$, there is

$$
\begin{aligned}
\alpha\left(\tau_{1} v\right) & =\int_{t_{1}}^{b}\left(\tau_{1} v\right)(t) \exp (-\lambda t) d t \\
& =\int_{t_{1}}^{b} \exp (-\lambda t)\left(\int_{a}^{t} h(s) f\left(s, \exp (\lambda s)\left(c_{1}(v)+\int_{s}^{b} v(\omega) \exp (-\lambda \omega) d \omega\right)\right) d s\right) d t \\
& \geq \frac{r_{1}}{m_{1}} \int_{t_{1}}^{b} \exp (-\lambda t)\left(\int_{a}^{t} h(\mathrm{~s}) d s\right) d t=r_{1} .
\end{aligned}
$$

Finally, we assert that $\beta\left(\tau_{1} v\right) \leq L_{1}$, in Lemma 1.1, also holds. If $v \in \partial p_{1}\left(\beta, L_{1}\right)$, by Lemma 2.3, for $t \in\left[t_{1}, b\right]$,

$$
\begin{aligned}
u(t) & =\exp (\lambda t)\left(c_{1}(v)+\int_{t}^{b} v(\omega) \exp (-\lambda \omega) d \omega\right) \\
& \geq v\left(t_{1}\right) \exp \left(\lambda t_{1}\right) \frac{\delta+\sum_{i=1}^{n} a_{i}\left(b-t_{i}\right) \exp \left(\lambda\left(t_{i}-b\right)\right)}{d_{1}} \\
& =L_{1} \exp \left(\lambda t_{1}\right) \frac{\delta+\sum_{i=1}^{n} a_{i}\left(b-t_{i}\right) \exp \left(\lambda\left(t_{i}-b\right)\right)}{d_{1}}
\end{aligned}
$$


The assumption $\left(\mathrm{A}_{3}\right)$ and (2.30) imply that

$$
\begin{aligned}
\beta\left(\tau_{1} v\right) & =\left(\tau_{1} v\right)(b) \\
& =\int_{a}^{b} h(s) f\left(s, \exp (\lambda s)\left(c_{1}(v)+\int_{s}^{b} v(\omega) \exp (-\lambda \omega) d \omega\right)\right) d s \\
& \leq \frac{L_{1}}{M_{1}} \int_{a}^{b} h(s) d s=L_{1} .
\end{aligned}
$$

To sum up, the hypotheses of Lemma 1.1 are satisfied. Hence, the operator $\tau_{1}$ has at least one fixed point, that is, the problem (1.4), (1.5) has at least one positive solution.

Let the cone

$$
D_{2}=\{v \in \mathbb{B}: v \text { is nonincreasing on }[a, b], v(b)=0\} .
$$

Evidently, $p_{2} \subset \boldsymbol{B}$. For $v \in p_{2}$, define the operator $\tau_{2}$ by

$$
\tau_{2} v(t)=\int_{t}^{b} h(s) f\left(s, \exp (\lambda s)\left(c_{2}(v)+\int_{a}^{s} v(\omega) \exp (-\lambda \omega) d \omega\right)\right) d s, \quad t \in[a, b],
$$

where $c_{2}(v)=\left(1 / d_{2}\right)\left(\delta v(a)-\sum_{i=1}^{n} a_{i} \exp \left(\lambda t_{i}\right) \int_{a}^{t_{i}} v(\omega) \exp (-\lambda \omega) d \omega\right)$.

We only give the preliminary lemmas and result of the problem (1.4), (1.6), the proofs are similar to the above argument.

Lemma 2.6. If $v \in P_{2}$ is a fixed-point $\tau_{2}$, then

$$
u(t)=\exp (\lambda t)\left(c_{2}(v)+\int_{a}^{t} v(\omega) \exp (-\lambda \omega) d \omega\right)
$$

is one solution of the problem (1.4), (1.6).

For $v \in D_{2}$, the nonnegative continuous functionals $\alpha$ and $\beta$ on $p_{2}$ are defined by

$$
\alpha(v)=\int_{a}^{t_{n}} v(\omega) \exp (-\lambda \omega) d \omega, \quad \beta(v)=v\left(t_{n}\right)
$$

Lemma 2.7. Let $r_{2}>0$. If $v \in \partial p_{2}\left(\alpha, r_{2}\right)$, then

$$
v\left(t_{n}\right) \leq \frac{\exp \left(\lambda t_{n}\right)}{t_{n}-a} r_{2}, \quad \int_{a}^{b} v(\omega) \exp (-\lambda \omega) d \omega \geq r_{2}
$$


Lemma 2.8. Let $L_{2}>0$. If $v \in \partial p_{2}\left(\beta, L_{2}\right)$, then

$$
\int_{a}^{t_{n}} v(\omega) \exp (-\lambda \omega) d \omega \geq L_{2} \exp \left(-\lambda t_{n}\right)\left(t_{n}-a\right)
$$

Lemma 2.9. Let $\left(H_{1}\right)-\left(H_{4}\right)$ hold, then $\tau_{2}: p_{2} \rightarrow p_{2}$ is completely continuous.

For convenience, we set

$$
\begin{gathered}
m_{2}=\int_{a}^{t_{n}} \exp (-\lambda t)\left(\int_{t}^{b} h(s) d s\right) d t>0, \quad M_{2}=\int_{a}^{b} h(s) d s>0, \\
u_{3}=r_{2} \exp (\lambda b), \quad u_{4}=L_{2} \exp (\lambda a) \frac{\delta-\sum_{i=1}^{n} a_{i}\left(t_{i}-a\right) \exp \left(\lambda\left(t_{i}-a\right)\right)}{d_{2}} .
\end{gathered}
$$

Theorem 2.10. Suppose that $\left(H_{1}\right)-\left(H_{4}\right)$ hold. Assume that $\delta-\sum_{i=1}^{n} a_{i} \exp \left(\lambda\left(t_{i}-a\right)\right)\left(t_{i}-a\right)>0$, then there exist positive numbers $k_{2}, r_{2}$, and $L_{2}$ with $\left(\left(\exp \left(\lambda t_{n}\right)\right) /\left(t_{n}-a\right)\right) r_{2}<L_{2}$ such that

$\left(\mathrm{B}_{1}\right) f(t, w) \geq k_{2},(t, w) \in[a, b] \times\left[r_{2}, \infty[\right.$,

(B) $f(t, w) \geq\left(r_{2} / m_{2}\right),(t, w) \in\left[t_{n}, b\right] \times\left[u_{3}, \infty[\right.$,

(B $\left.\mathrm{B}_{3}\right) f(t, w) \leq\left(L_{2} / M_{2}\right),(t, w) \in\left[a, t_{n}\right] \times\left[u_{4}, \infty[\right.$.

Then the operator $\tau_{2}$ has at least one fixed-point $v$ such that $r_{2} \leq \alpha(v)$ and $\beta(v) \leq L_{2}$, and the problem (1.4), (1.6) has at least one positive solution u such that

$$
u(t)=\exp (\lambda t)\left(c_{2}(v)+\int_{a}^{t} v(\omega) \exp (-\lambda \omega) d \omega\right)
$$

\section{Examples}

Consider the problems

$$
\begin{aligned}
& u^{\prime \prime}(t)-u^{\prime}(t)+h(t) f(t, u(t))=0, \quad 0<t<1, \\
& u^{\prime}(0)-u(0)=0, \quad u(1)+2 u^{\prime}(1)=2 u\left(\frac{1}{2}\right), \\
& u(0)+2 u^{\prime}(0)=2 u\left(\frac{1}{2}\right), \quad u^{\prime}(1)-u(1)=0 .
\end{aligned}
$$

Let

$$
h(t)=\frac{1}{\sqrt{t(1-t)}}, \quad f(t, w)= \begin{cases}10^{-3} t+\frac{2}{\sqrt{w}+2}, & w<2 \\ 10^{-3} t+2-\sqrt{2}, & w \geq 2\end{cases}
$$


It is easy to check that hypotheses $\left(\mathrm{H}_{1}\right)-\left(\mathrm{H}_{4}\right)$ hold. For the problem (3.1), (3.2), by some calculations, we have $d_{1} \approx 4.858, m_{1} \approx 0.348$, and $M_{1} \approx 3.142$. Taking $k_{1}=0.001, r_{1}=0.2$, and $L_{1}=2$, satisfying the following conditions: $f(t, w) \geq 0.001,(t, w) \in[0,1] \times[0.2, \infty[, f(t, w) \geq$ $r_{1} / m_{1} \approx 0.575,(t, w) \in[0,1 / 2] \times\left[0.141, \infty\left[, f(t, w) \leq L_{1} / M_{1} \approx 0.637\right.\right.$, and $(t, w) \in[1 / 2,1] \times$ $\left[2.242, \infty\right.$ [. Thus, the hypotheses of Lemma 1.1 are fulfilled, and so the operator $\tau_{1}$ has at least one fixed point, that is to say, the problem (3.1), (3.2) has at least one positive solution. For the problem (3.1), (3.3), by some calculations, we obtain $d_{2} \approx 0.297, m_{2} \approx 0.348$, and $M_{2} \approx 3.142$. Taking $k_{2}=0.001, r_{2}=0.1$, and $L_{2}=2$, combining with the following conditions: $f(t, w) \geq 0.001,(t, w) \in[0,1] \times\left[0.1, \infty\left[, f(t, w) \geq r_{2} / m_{2} \approx 0.287,(t, w) \in[1 / 2,1] \times[0.272, \infty[\right.\right.$, $f(t, w) \leq L_{2} / M_{2} \approx 0.637$, and $(t, w) \in[0,1 / 2] \times[2.364, \infty[$. So the problem (3.1), (3.3) has at least one positive solution.

\section{References}

[1] V. A. I1'in and E. I. Moiseev, "Nonlocal boundary value problem of the second kind for a SturmLiouville operator," Differential Equations, vol. 23, pp. 979-987, 1987.

[2] A. V. Bitsadze, "On the theory of nonlocal boundary value problems," Soviet Mathematics Doklady, vol. 30, pp. 8-10, 1984.

[3] A. V. Bitsadze, "On a class of conditionally solvable nonlocal boundary value problems for harmonic functions," Soviet Mathematics Doklady, vol. 31, pp. 91-94, 1985.

[4] A. V. Bitsadze and A. A. Samarski1,, "On some simple generalizations of linear elliptic boundary value problems," Soviet Mathematics Doklady, vol. 10, pp. 398-400, 1969.

[5] C. P. Gupta, "Solvability of a three-point nonlinear boundary value problem for a second order ordinary differential equation," Journal of Mathematical Analysis and Applications, vol. 168, no. 2, pp. 540-551, 1992.

[6] C. P. Gupta, S. K. Ntouyas, and P. Ch. Tsamatos, "On an $m$-point boundary-value problem for secondorder ordinary differential equations," Nonlinear Analysis: Theory, Methods \& Applications, vol. 23, no. 11, pp. 1427-1436, 1994.

[7] C. P. Gupta and S. I. Trofimchuk, "Existence of a solution of a three-point boundary value problem and the spectral radius of a related linear operator," Nonlinear Analysis: Theory, Methods \& Applications, vol. 34, no. 4, pp. 489-507, 1998.

[8] N. Anderson and A. M. Arthurs, "Analytical bounding functions for diffusion problems with Michaelis-Menten kinetics," Bulletin of Mathematical Biology, vol. 47, no. 1, pp. 145-153, 1985.

[9] D. Aronson, M. G. Crandall, and L. A. Peletier, "Stabilization of solutions of a degenerate nonlinear diffusion problem," Nonlinear Analysis: Theory, Methods \& Applications, vol. 6, no. 10, pp. 1001-1022, 1982.

[10] A. Nachman and A. Callegari, "A nonlinear singular boundary value problem in the theory of pseudoplastic fluids," SIAM Journal on Applied Mathematics, vol. 38, no. 2, pp. 275-281, 1980.

[11] R. P. Agarwal and D. O'Regan, Singular Differential and Integral Equations with Applications, Kluwer Academic Publishers, 2003.

[12] H. Asakawa, "Nonresonant singular two-point boundary value problems," Nonlinear Analysis: Theory, Methods E Applications, vol. 44, pp. 791-809, 2001.

[13] R. Ma and D. O’Regan, "Solvability of singular second order $m$-point boundary value problems," Journal of Mathematical Analysis and Applications, vol. 301, no. 1, pp. 124-134, 2005.

[14] D. O'Regan, Theory of Singular Boundary Value Problems, World Scientific, River Edge, NJ, USA, 1994.

[15] Q. Yao, "Existence and iteration of $n$ symmetric positive solutions for a singular two-point boundary value problem," Computers \& Mathematics with Applications, vol. 47, no. 8-9, pp. 1195-1200, 2004.

[16] C. De Coster and P. Habets, "Upper and lower solutions in the theory of ODE boundary value problems: classical and recent results," in Recherches de Mathématique, vol. 52, pp. 1-78, Institut de Mathématique Pure et Appliquée, Université Catholique de Louvain, 1996.

[17] J. Mawhin, Topological Degree Methods in Nonlinear Boundary Value Problems, vol. 40 of NSFCCBMS Regional Conference Series in Mathematics, American Mathematical Society, Providence, RI, USA, 1979. 
[18] R. W. Leggett and L. R. Williams, "Multiple positive fixed points of nonlinear operators on ordered Banach spaces," Indiana University Mathematics Journal, vol. 28, no. 4, pp. 673-688, 1979.

[19] Y. Zhang, "Positive solutions of singular sublinear Emden-Fowler boundary value problems," Journal of Mathematical Analysis and Applications, vol. 185, no. 1, pp. 215-222, 1994.

[20] R. I. Avery and D. R. Anderson, "Fixed point theorem of cone expansion and compression of functional type," Journal of Difference Equations and Applications, vol. 8, no. 11, pp. 1073-1083, 2002. 


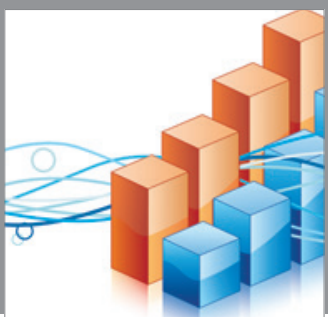

Advances in

Operations Research

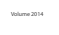

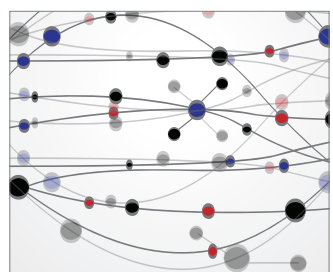

\section{The Scientific} World Journal
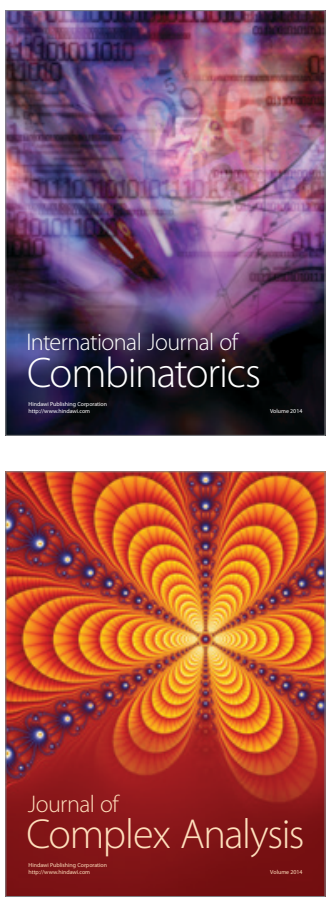

International Journal of

Mathematics and

Mathematical

Sciences
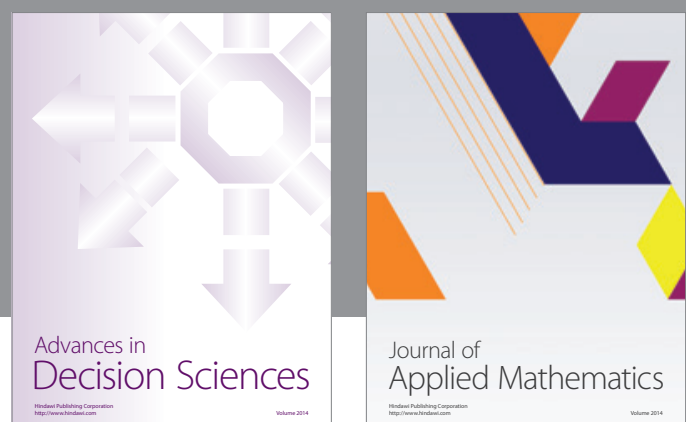

Journal of

Applied Mathematics
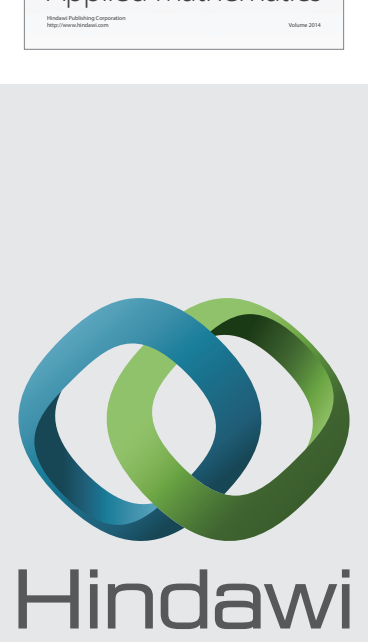

Submit your manuscripts at http://www.hindawi.com
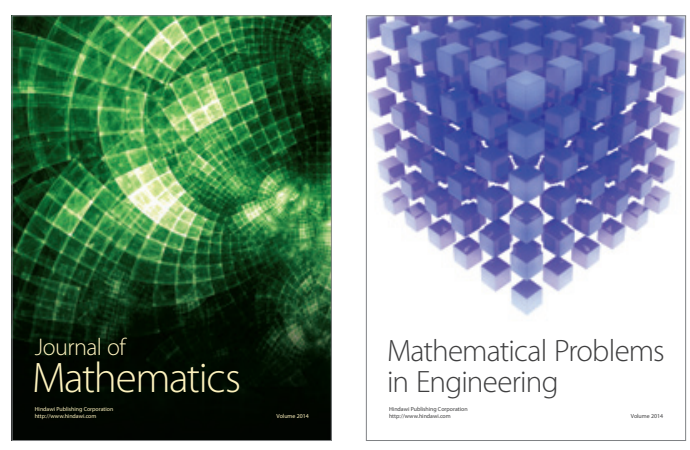

Mathematical Problems in Engineering
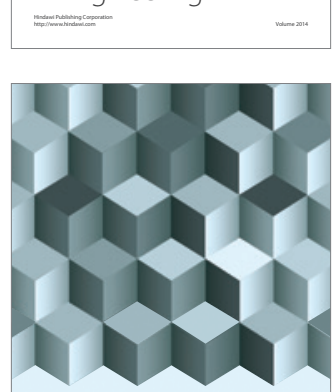

Journal of

Function Spaces
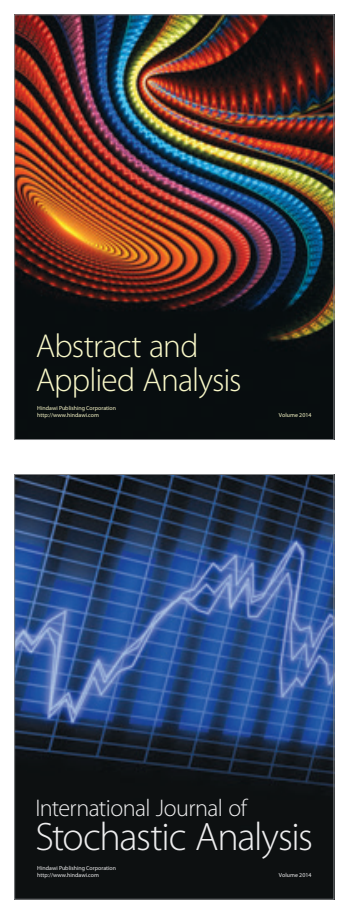

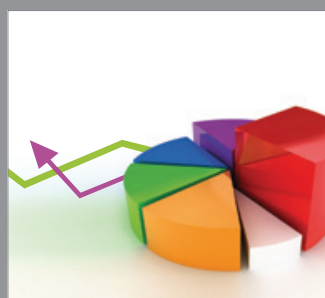

ournal of

Probability and Statistics

Promensencen
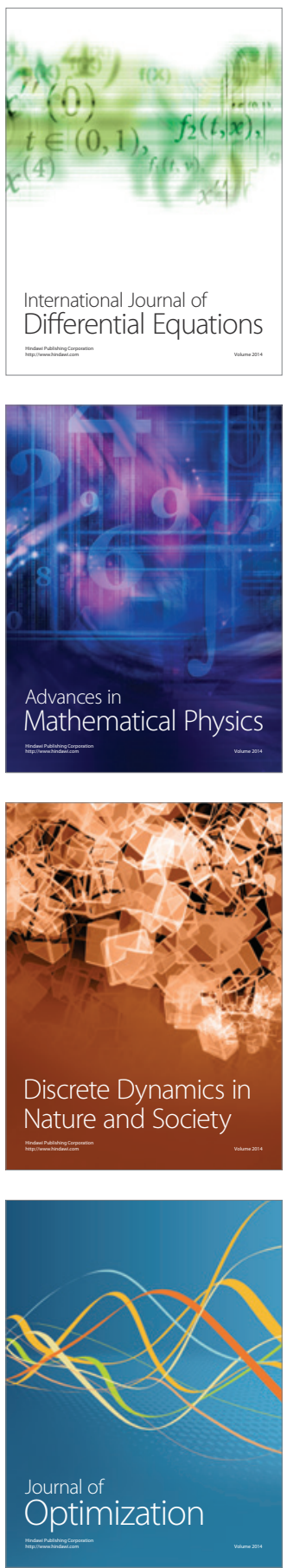